\title{
Venous System \\ Interventions for Device Implantation
}

\author{
Jose M. Marcial, MD, Seth J. Worley, MD, FHRS*
}

\section{KEYWORDS}

- Subclavian occlusion • Venoplasty • Upgrade • Biventricular • Occlusion • Stenosis • His pacing

- Focused force venoplasty

\section{KEY POINTS}

- The 2017 Heart Rhythm Society (HRS) Expert Consensus Statement recognizes subclavian venoplasty (SV) as a safe and effective lead management option when venous access becomes an issue due to occlusion of the desired access point.

- Peripheral vein venography, sonography, CT scan, and so forth overestimate the severity of the obstruction. Contrast injection at the site of occlusion (local venogram) frequently delineates a path to the central circulation.

- Compared with progressively larger dilators, SV improves the quality of venous access, providing the unrestricted catheter manipulation critical to both His bundle pacing and left ventricular lead implantation.

- SV preserves venous access and reduces lead burden by decreasing the need to tunnel or implant a new system on the contralateral side.

- By acquiring the tools and following a consistent step-by-step approach, implanting physicians can easily add SV to lead management options.

Video content accompanies this article at http://www.cardiacep.theclinics.com.

\section{INTRODUCTION}

Subclavian vein stenosis/occlusion is an important clinical problem. As indications for cardiovascular implantable electronic devices (CIEDs) expand and patients with existing CIED leads require the addition or replacement of leads, implanting physicians increasingly need to navigate these venous occlusions. ${ }^{1-8}$ Moreover, subclavian obstructions can significantly impair the possibilities of achieving cardiac resynchronization by preventing placement of a left ventricular (LV) lead. ${ }^{9,10}$ Even if vascular access is gained using progressively larger dilators, catheter manipulation is

Conflict of Interest: None (J.M. Marcial). Royalties from Merit Medical and Pressure Products for the sale of the Worley LV lead implant tools. Compensation from Medtronic, Abbott, and Biotronik for teaching the "Interventional Approach to LV lead Implantation" using the Worley tools and techniques. Neither Merit Medical nor Pressure products sell a balloon for venoplasty. The author does not receive direct or indirect compensation from Merit Medical for the sale of the accessories (catheters, wires, and contrast injection system) discussed in this article. The author has no overt or covert financial interest in promoting the use of subclavian venoplasty to obtain and/or improve venous access (S.J. Worley).

Department of Medicine, Division of Cardiology, Cardiac Arrhythmia Center, Medstar Heart and Vascular Institute, Medstar Washington Hospital Center, 110 Irving Street Northwest, Washington, DC 20010, USA

* Corresponding author. Department of Medicine, Division of Cardiology, Cardiac Arrhythmia Center, Medstar Heart and Vascular Institute, Medstar Washington Hospital Center, 110 Irving Street Northwest, Suite 5A-12, Washington, DC 20010.

E-mail address: seth@mcworley.com 
frequently impaired by the residual stenosis. Restricted catheter manipulation impairs LV lead placement and His bundle pacing. Crossing intraluminal occlusions followed by subclavian venoplasty (SV) has been demonstrated a safe alternative. ${ }^{11-16}$ Furthermore, SV may be preferable to using a contralateral access approach, the supraclavicular approach, ${ }^{17,18}$ or powered/ mechanical sheath extraction, with extraction posing additional risks. ${ }^{19-23}$ The technique of SV should thus become an integral part of the core armamentarium of the implanting physician.

\section{EPIDEMIOLOGY}

The incidence of subclavian vein stenosis after device implantations varies widely in the literature, ranging from $30 \%$ to $50 \%$. $^{1,2,6,24-26}$ One group prospectively studied venography at 6 months postimplantation in 202 patients and identified greater than moderate stenosis in $51 \% .{ }^{27}$ Previous use of transvenous temporary leads, LV ejection fraction less than $40 \%$, and advanced age (>65 years) were found to be independent risk factors for a higher incidence of venous occlusion. Another group performed consecutive venography on 40 patients and found significant stenosis in $23 \%$ at a mean postprocedure follow-up of 4 months. ${ }^{25}$ Most of the patients in these studies were asymptomatic. The lack of symptoms was felt to be secondary to the collateral venous circulation that developed as the stenosis progressed.

\section{PATHOPHYSIOLOGY OF SUBCLAVIAN OCCLUSIONS}

Studies have described subclavian venous thrombosis of both an early ${ }^{25}$ (within 6 months) and late $^{26}$ (at 6 years) progression. Late thrombosis is most often asymptomatic and is a result of chronic fibrosis around the device leads, which allows time for concurrent collateralization. Autopsies have demonstrated inflammatory and fibrotic changes that occur at the endothelial-lead interface and at the insertion site of the leads in the vein. ${ }^{28}$ Dense fibrotic lesions are most likely to be found in anatomic sites like the lead insertion point, venous bifurcation sites, and the costoclavicular space. In a large series of more than 300 patients who underwent SV, obstructions were seen to be peripheral (subclavian/distal innominate) in 61\%, central (innominate/superior vena cava [SVC]) in $17 \%$, and both central and peripheral in $22.1 \%$ of cases. $^{12}$ Endothelial injury and repetitive mechanical trauma at bifurcation sites or where bony compression occurs may account for these findings.

\section{SAFETY AND EFFICACY OF SUBCLAVIAN VENOPLASTY}

The largest series reporting the use of SV for lead implantation included 373 cases over an 11-year period. ${ }^{12}$ Successful access was achieved in 371 of 373 , with no adverse clinical outcome, no distal embolization, no venous disruption, and no acute damage to the leads. The same group subsequently extended the results to $488 \mathrm{SV}$ procedures performed from January 27, 2004, to November 20, 2014, via an electronic health record query. There were no acute clinical events in the cohort. Contrast extravasation was common during crossing of total occlusions and was observed with balloon ruptures on 3 occasions, but none was clinically significant. Questioning whether the pressure and mechanical stress to which existing leads are subjected during SV affect long-term lead performance, the group also looked for damage to existing leads becoming manifest over 12 months after venoplasty. Of the initial 488 patients, 20 were lost to follow-up. In the remaining 468 patients, there were 2 atrial lead dislodgements and 1 insulation defect found in a preexisting atrial lead. The atrial lead dislodgements were detected at 3 months and 5 months post SV, respectively, whereas the insulation defect was detected at 9 months. When compared with a cohort who had lead replacement without SV, there was no evidence to suggest that SV caused delayed lead damage. In an attempt to acquire additional safety data, an email survey of 23 centers performing SV was conducted in 2015. All centers contacted responded. There was a mean of 19.4 cases \pm 12.7 cases per center with no center reporting an adverse event. Thus, it seems that SV not only is safe but also does not pose any danger to existing leads.

Operative lead management should include SV as a means to conserve venous access and assist in optimal lead placement. Because of the extensive fibrous tissue surrounding the leads (Fig. 1), venoplasty is an intrinsically safe

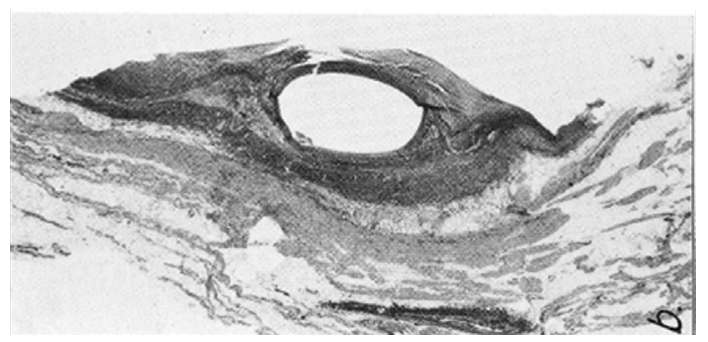

Fig. 1. Example of the dense fibrous tissue that develops around the pacing lead. 
procedure and should be considered fibroplasty as opposed to venoplasty. The safety experience described by Worley and colleagues, ${ }^{12}$ however, applies to patients with chronic leads ( $>2$ years) where venoplasty was performed to add a lead(s). When SV is compared with the use of progressively larger dilators, venoplasty is faster and there are problems with dilators: catheters remain difficult to manipulate throughout the procedure, the more central stenosis at the SVC-right atrium (RA) junction is not dilated, and a false sense of safety is created. There are some situations where venoplasty may not be advisable, such as at eccentric occlusions along the SVC (Fig. 2) and for a swollen arm developing within the first several months of implant, indicative of early progression of thrombosis. In the latter situation, fibrous tissue has not yet developed and larger balloons are required, increasing the likelihood of venous disruption. In addition, there is little evidence that venoplasty for a swollen arm produces lasting patency, and spontaneous collateral vein development usually results in long-term clinical resolution of arm swelling. Spittel and colleagues ${ }^{3}$ reported early success with venoplasty in the treatment of 2 patients with symptomatic venous stenosis. In view of these risks, however, conservative options, including watchful waiting for collateral vessels to develop, seems a reasonable alternative.

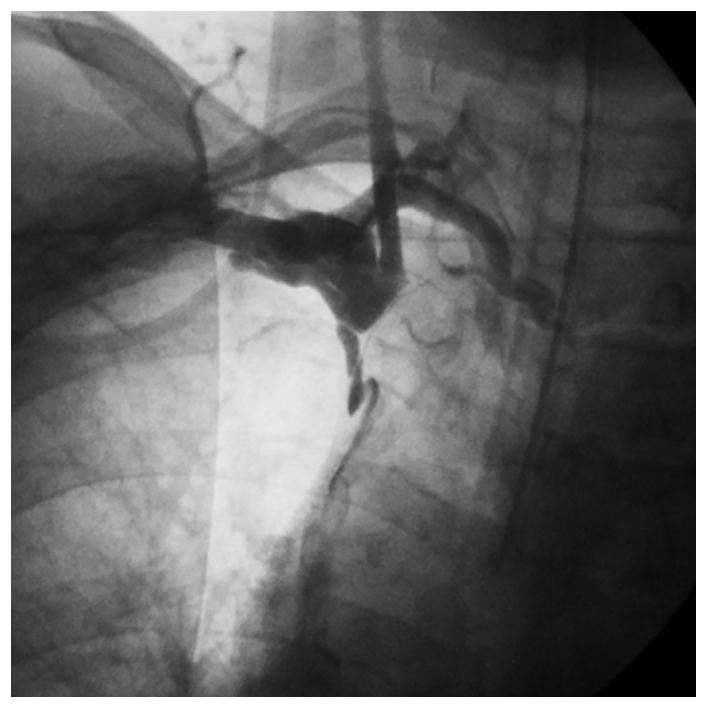

Fig. 2. The predominant area of obstruction is along the lateral wall SVC rather than within the subclavian innominate vein. Although a wire was easily advanced into the RA, venoplasty was not performed because of the lack tissue surrounding the obstruction and the catastrophic consequences of any SVC tear.

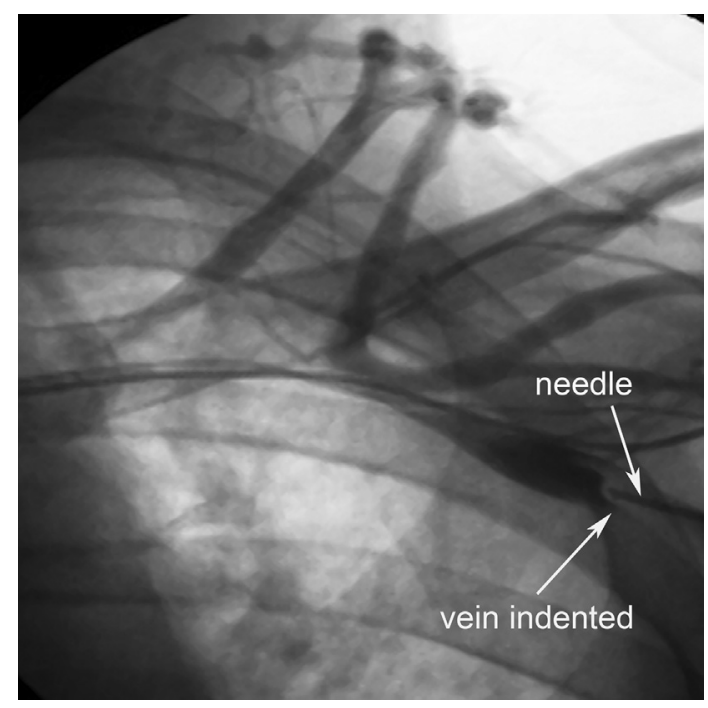

Fig. 3. Venous access as contrast is injected into a peripheral vein. The occlusion along with collateral veins are apparent. The vein is entered peripheral to the occlusion. When the needle is inserted into the vein as contrast is flowing, the vein can be seen to indent, confirming where the needle enters the vein. When crossing an occlusion, it is important to enter the vein as far peripheral to the occlusion as possible.

$\mathrm{Ji}$ and colleagues ${ }^{23}$ were able to evaluate the utility of SV at their hospital, wherein only some of the implanting physicians decided to learn and adopt the SV technique; 41 patients with subclavian vein occlusion were scheduled for the addition of a lead(s); implanters who adopted the SV technique performed 18 cases and implanters who did not adopt the SV technique performed 23 cases. When implanting physicians were competent in the venoplasty technique, procedure times were shorter (2:31 hours for the venoplasty competent operators compared with 3:28 hours for operators who were not venoplasty competent). When the implanting physicians were venoplasty competent, implants were more frequently successful: there were 5 implant failures in the 23 cases $(21.5 \%)$ resulting from inability to gain venous access when the implanter was not venoplasty competent. On the other hand, there were no implant failures in the 18 cases when the implanter was venoplasty competent. ${ }^{23}$

\section{Step-by-Step Approach to Subclavian Venoplasty}




\section{Steps Description \\ Step 1 In patients with previous leads, because of the possibility of subclavian obstruction, the authors start venous access with a $15-\mathrm{cm}$ stiffened dilator micropuncture kit (Box 1) rather than using the 18-gauge needle and wire that come with the sheath.}

Step 2 Perform peripheral venogram using a routine intravenous line. Although the contrast is flowing, enter the vein with the needle as far peripheral to the occlusion as possible (Fig. 3; Videos 1-3) without regard to the location of the rib. Unlike traditional axillary vein access, the needle should enter the body at a shallow angle of approximately $30^{\circ}$.
The key to success is to enter the vein peripheral to the occlusion at a shallow angle ( $30^{\circ}$ to the skin). To this end, it is best to puncture the vein while contrast is flowing to confirm where the needle enters the vein (see Videos 1-3). To achieve the proper angle and vein entry site, it is usually necessary to insert the needle into the skin lateral to the pocket and tunnel the wire into the pocket. If the needle enters the vein at the site of occlusion, it is difficult to advance the wire and any opening through which to advance the wire will be lost. Do not be concerned if it seems like there is a total occlusion, the peripheral venogram usually overestimates the severity of the obstruction (Fig. 4, Video 4).

\section{Step 3 Carefully advance the wire into the vein up to the site of obstruction. It is important to not disrupt the site of obstruction with the needle or the wire.}

Step 4 Carefully advance the $5 \mathrm{~F}$ dilator or stiffened dilator $/ 5 \mathrm{~F}$ catheter over the wire (see Videos 1 and 2; Video 5). The tip of the dilator should not be pushed beyond the tip of the wire. The goal is the get the tip of the dilator/catheter a few millimeters peripheral to the obstruction.

Step 5 Attach a contrast injection system with hemostatic Y-adapter to the hub of the dilator/catheter (Figs. 5 and 6). An injection system similar to that shown in Fig. 5A can be assembled from spare parts available in most laboratories or purchased more cost effectively as a kit (see Box 1).

Step 6 Insert a 0.035 -in or 0.018-in nitinol angled polymer tip hydrophilic wire (glide wire) into the hemostatic valve. Nitinol angled polymer tip hydrophilic wires are available from several vendors (see Box 1). In some cases, the 0.035 -in wire is too large to cross the obstruction.
The 0.018-in nitinol polymer tip hydrophilic wire is superior to a 0.014 -in angioplasty wire because the angioplasty wire is stainless steel, which is easily bent whereas the nitinol 0.018-in wire retains its shape.

Step 7 Attach a torque device to the proximal end of the wire $5 \mathrm{~cm}-10 \mathrm{~cm}$ from where it enters the hemostatic valve (see Fig. 5C).

Step 8 Close the hemostatic valve.

Step 9 Use puffs of contrast and the torque device to In many cases the glide wire crosses the direct the wire across the occlusion (see Videos 1 and 5). occlusion. If not the $5 \mathrm{~F}$ dilator/micropuncture catheter is replaced with a braided angled hydrophilic catheter (see Box 1) to provide direction and support for the wire. 
(continued)

\section{Steps Description}

Step 10 If the wire alone cannot be advanced across the obstruction, replace the $5 \mathrm{~F}$ dilator $/ 5 \mathrm{~F}$ micropuncture catheter with an angled 4 or $5 \mathrm{~F}$ braided catheter with hydrophilic coating (see Box 1).
Step 11 When a wire cannot be advanced through the occlusion, retained wire lead removal followed by venoplasty is an option if one of the leads is movable within the fibrous adhesions (Video 7). Retained wire lead removal can be accomplished via (1) wire under the insulation technique, (2) wire in the stylet lumen with femoral removal, (3) over the wire removal of an LV pacing lead.

Step 12 Confirm that the wire has entered the heart. Advance the wire until the tip enters either the pulmonary artery or the inferior vena cava. If the wire does not advance into the pulmonary artery or inferior vena cava, do not proceed.

Step 13 Exchange the glide wire for an Amplatz Extra Stiff wire using a braided 4 or $5 \mathrm{~F}$ hydrophilic catheter.

\section{Additional Comments}

As detailed in Box 1, the angle and length of the tip as well as the braid in the walls of the catheter are critical, and substitution is not advised. The angled-tip catheter is used to inject contrast and aim the wire toward an opening (see Video 2). A variety of hydrophilic exchange catheters are available but a braided catheter is preferred because of greater pushability and torque capability compared with nonbraided catheters (eg, Terumo Glidecath). The catheter is stiff enough to be pushed into the occlusion followed by the wire (see Video 3). On occasion, advancing the catheter with puffs of contrast without a wire can be more effective than blindly trying to follow a wire (Video 6).

\section{Confirm in the right anterior oblique and left anterior oblique projections that the wire follows the lead(s) to the SVC or PA.}

The glide wire used to cross the occlusion often does not provide sufficient support to advance the balloon through the obstruction (see Video 4).

Step 14 Advance a $6-\mathrm{mm}$ or $9-\mathrm{mm} \times 4-\mathrm{cm}$ ultranoncompliant balloon (lumen diameter .035-in) over the Amplatz Extra Stiff wire (see Box 1) to just beyond the SVCinnominate junction. Use a 9-mm balloon if the plan is to add 2 leads or there is elastic recoil after 6-mm balloon inflation.

Step 15 If the ultranoncompliant balloon does not track to the SVC-innominate junction, first try predilating with the lower profile 4-mm $\times 4$-cm noncompliant balloon (see Box 1). If the 4-mm balloon does not advance, move to a .018-in lumen lower profile balloon using the braided catheter to replace the 0.035-in Amplatz wire with a 0.018-in extrastiff wire (eg, V-18 Control Wire). Predilate with the .018-in lumen low-profile balloon (eg, Sterling Balloon). Once dilated, use the catheter to replace the .018-in wire with the .035-in Amplatz wire and finish dilating with the $6-\mathrm{mm}$ or $9-\mathrm{mm}$ ultranoncompliant balloon.

If the 4-mm balloon does not advance, switch to a .018-in lumen lower-profile balloon using the braided catheter to replace the 0.035-in Amplatz wire with a 0.018-in extrastiff wire (eg, V-18 Control Wire). Predilate with the .018-in lumen low-profile balloon (eg, Sterling Balloon). Once dilated, use the catheter to replace the .018-in wire with the .035-in Amplatz wire and finish dilating with the $6-\mathrm{mm}$ or $9-\mathrm{mm}$ ultranoncompliant balloon 


\begin{tabular}{|c|c|}
\hline Steps & Description \\
\hline Step 16 & $\begin{array}{l}\text { Always perform the first inflation at or central } \\
\text { to the SVC-innominate junction because a } \\
\text { stenosis is often present but not recognized } \\
\text { on the venogram. } \\
\text { The profile of the balloon increases after the } \\
\text { first inflation-deflation cycle (called } \\
\text { winging), making it impossible to advance } \\
\text { to the SVC-innominate junction. }\end{array}$ \\
\hline
\end{tabular}

Step 17 Inflate the balloon to the rated burst pressure (RBP) indicated on the balloon package (26 atm-30 atm for the peripheral balloons listed in Box 1 and 18 atm-20 atm for the low-profile peripheral balloon (ie, Sterling Balloon).

\section{Additional Comments}

Initially, the authors did not dilate at the SVCinnominate junction but when advancing the sheath was tried, a stenosis requiring additional venoplasty was found in approximately $20 \%$ of cases. Because the profile of the balloon increases after the first inflation-deflation cycle (called winging), the balloon used for the more apparent peripheral stenosis did not advance through the central stenosis.

Step 18 Keep the balloon inflated until the pressure is stable at RBP and there is no residual waist. If the waist is not eliminated, use focused force venoplasty (Fig. 7).

Step 19 Deflate the balloon by applying negative pressure to the inflation device.

Step 20 Once the contrast is evacuated from the balloon, withdraw the balloon until the tip reaches the former tail position (head to tail overlap).

Do not withdraw the balloon until the contrast is evacuated from the balloon (Fig. 8).

Step 21 Continue overlapping inflations until the tail The recommendation to continue until the of the balloon is visible in the pocket.

tail is visible in the pocket frequently produces concern but is essential and does not seem to cause excessive bleeding. If bleeding is a problem, it is easily addressed with a hemostatic suture.

Step 22 Advance a long $(25-\mathrm{cm})$ sheath over the wire. The other explanation for difficulty Always use a long sheath after venoplasty. If advancing the sheath is a residual peripheral stenosis from failure to inflate the balloon with the tail visible in the it is not very easy to advance the sheaths, there is excessive elastic recoil. If elastic recoil is present, it is worth taking the time to upsize to a 9-mm diameter balloon. pocket as recommended.

Step 23 If 2 leads are needed, advance 2 Amplatz Extra Stiff wires into the long sheath and withdraw the sheath retaining the 2 wires.

\section{BALLOON OPTIONS}

Coronary balloons are too small, in both length and diameter, for SV. With a 0.035-in wire across the obstruction, it makes sense to start with a .035-in lumen balloon (see Box 1). With retained wire lead removal and when the stenosis is tight enough to require a 0.018 -in wire, predilate with a .018-in lumen ultra-low-profile balloon (eg, Sterling). As indicated in Box 1, the recommended balloon diameter is $6 \mathrm{~mm}$ or
$9 \mathrm{~mm}$ and length $4 \mathrm{~cm}$ to $6 \mathrm{~cm}$. The $9-\mathrm{mm}$ balloon is useful if there is excess elastic recoil or if the intent is to add 2 leads. Alternatively, elastic recoil may be addressed with focused force venoplasty (see Fig. 7). If a 9-mm diameter balloon is not available for adding 2 leads, the stenosis can be redilated with the $6-\mathrm{mm}$ balloon when the first long sheath (with dilator) is in place. The recommended balloons are $75 \mathrm{~cm}$ to $80 \mathrm{~cm}$ in length, thus requiring only a standard-length wire. 
Box 1

Annotated list of equipment for crossing and dilating a subclavian obstruction/occlusion

Contrast injection system

Attached the dilator from a $5 \mathrm{~F}$ sheath or the $5 \mathrm{~F}$ catheter of the micropuncture system. Contrast injection with the tip of the dilator/catheter at the site of occlusion often identifies an opening through which to advance a wire. The $\mathrm{Y}$-adapter with rotating hemostatic valve allows the wire to be advanced through the dilator/catheter to cross the occlusion: contrast injection system Worley Advanced Kit 1 CAK 1 (comes with contrast bowl and labels) (order \# K12-WORLEY1, Merit Medical)

Dilator from a $5 \mathrm{~F}$ sheath vs stiffened micropuncture

Prior adopting the micropuncture kit with stiffened radiopaque dilator, the dilator from $5 \mathrm{~F}$ sheath was advanced over the 0.035 -in J wire. Conversion to the stiffened micropuncture kit was precipitated by

1. Difficulty advancing a 0.035 -in wire into the vein for initial venous access (easier using a 0.018 -in wire)

2. Difficulty advancing the dilator over the wire (not stiff enough)

3. Difficulty visualizing the tip of the dilator to be certain it was in the vein (not radiopaque)

The authors now use 5F 15-cm micropuncture kit with stiffened radiopaque dilator for initial venous access. The 21-gauge needle and angled-tip 0.018-in nitinol wire can make venous access easier. Compared with standard micropuncture, the stiffened dilator (indicated by the $S$ in the order number) provides the support required to advance over the wire through scar tissue. Being radiopaque makes it is easier to be certain the tip is in the vein. The $15-\mathrm{cm}$ length (usually $10 \mathrm{~cm}$ ) makes it more likely that the tip reaches beyond the stenosis. Catheter $=5 \mathrm{~F} \times 15-\mathrm{cm}$; wire $=0.018$-in $\times 60-\mathrm{cm}$ nitinol with platinum tip; needle $=21 \mathrm{G}$ (order \# S-MAK501N15BT)

Wires for crossing subclavian obstructions

Angled polymer jacketed nitinol wires with a hydrophilic coating (also known as glide wires) work well for crossing subclavian obstructions. These wires are available from multiple vendors with slightly different names. In general, 0.014-in angioplasty wires are not helpful because they are constructed from stainless steel, which is easily deformed whereas nitinol wires tend to retain their shape.

1. 0.035 -in $\times 180$-cm angled-tip (not straight) wires available from multiple vendors, including Merit Medical Laureate wire (order \# LWSTDA35180, Merit Medical)

2. 0.018 -in $\times 180-\mathrm{cm}$ angled-tip (not straight) wire available from multiple vendors, including Merit Medical Laureate wire (order \# LWSTDA18180, Merit Medical)

Wires for retained wire lead removal

For the wire under the insulation/wire in the stylet lumen and over the wire removal of most LV leads use a polymer jacketed extra support wire (eg, Choice PT Extra Support or Acuity Whisper Extra Distal Support [Boston Scientific])

Medtronic LV leads have a 0.018 -in lumen, which allows removal over a more supportive wire (eg, V-18 Control Wire (polymer jacketed 200-cm Boston Scientific).

Torque device (also known as steering handle) for directing the angled-tip glide wire

A torque device is used to direct the tip of glide wire (caution: 1 size may not work for all wires) (A) Torque device for 0.014 in-0.018-in wires. (B) Torque device for 0.025 in-0.038-in wires.

Catheters used to (1) exchange from glide to Amplatz Extra Stiff wire and (2) cross a difficult subclavian obstruction/total occlusion.

1. Catheters for wire exchange

The glide wire used to cross the occlusion frequently does not provide sufficient support to advance the balloon. The glide wire must be exchanged for an extra support wire (Amplatz Extra Support). For wire exchange, a 4F/5F braided catheter with hydrophilic coating works best. The braid and hydrophilic coating are essential. Similar catheters without metal braid (eg, Terumo Glidecath) may not have adequate stability to be advanced over the wire into the central circulation. Although the $5 \mathrm{~F}$ is most commonly used, occasionally a tight stenosis requires downsizing to 4F. An alternative is the straight catheter when the glide wire advances to the central circulation without the need for an angled-tip catheter. 


\section{Catheters for crossing difficult occlusions}

To cross a totally occluded subclavian, a properly shaped, angled-tip, braided catheter with a hydrophilic coating is essential. The length and angle of the tip are critical; slight variations are surprisingly important. The catheter is used in 2 ways: (1) to better direct and provide support for the glide wire; the braided catheter provides support to push the wire into the occlusion; once the wire advances the catheter is worked up to the tip of the wire, and (2) with puffs of contract without a wire to navigate through collaterals and tortuosity; I have the most experience with the $5 \mathrm{~F}$ version of the catheters listed; similar catheters without metal braid (eg, Terumo Glidecath) do not have adequate torque control or support:

1. $5 \mathrm{~F}$ Impress KA2 Hydrophilic Angiographic Catheter 5F 65-cm (order \# 56538KA2-H, Merit Medical)

2. 4F Impress KA2 Hydrophilic Angiographic Catheter 4F 65-cm (order \# 46538KA2-H, Merit Medical)

J-tip extra stiff support wire to replace the glide wire

Note: Not all Amplatz Extra Stiff wires are created equal! For example, both the Boston Scientific and Cook are labeled "Amplatz Extra Stiff"; the floppy distal section of the Boston Scientific J-tip Amplatz is too long to be useful for providing support in the coronary sinus. To advance a balloon, either wire is satisfactory; for simplicity of inventory, order the Cook J-tip Amplatz 0.035-in $\times 180$-cm (THSCF-35180-3-AES, order \# G03565).

Balloons for SV when a 0.035-in wire is advanced across the obstruction

A 0.035 -in glide wire is usually used to cross the obstruction, thus a balloon with a 0.035 -in lumen is required. If a $.014 / .018$-in wire is across the obstruction see balloons for retained wire lead removal below.

1. To add 1 lead, use a 6-mm diameter $\times 4-\mathrm{cm}$ balloon with a shaft length $75-\mathrm{cm}$. Example, CONQUEST (Kevlar), order \# CQ-7564, Bard Peripheral Vascular Ultra-Noncompliant: wire lumen 0.035-in, over the wire, RBP 30 atm.

2. To add 2 leads or if there is elastic recoil, use a $9-\mathrm{mm} \times 4-\mathrm{cm}$ balloon with a shaft length $75-\mathrm{cm}$ balloon, Bard Peripheral Vascular Ultra-Noncompliant: wire lumen 0.035-in, over the wire, RBP 26 atm. Order \# CQ-7594, Bard Peripheral Vascular.

3. When the balloons (discussed previously) do not advance across the obstruction predilate with (1) Cordis Powerflex Pro OTW Balloon (0.035-in wire lumen) 4-mm $\times 40-\mathrm{mm}$ balloon shaft length $80 \mathrm{~cm}$; order \# 44004045 or (2) exchange the 0.035 -in wire for 0.018 -in extra support wire (V-18 Control Wire) and predilate with a low profile peripheral balloon (eg, Sterling Balloon [discussed later]).

Balloons for SV when a 0.014 -in/0.018-in wire is advanced or introduced by the retained wire lead removal technique

If a .018-in lumen balloon is advanced over a .014-in wire, once the tip of the balloon is in the central circulation, the 0.0014 -in wire should be exchanged for a more supportive .018-in wire, for example, V-18 Control Wire (discussed previously). To adequately dilate the obstruction, the 0.018 -in lumen balloon frequently needs to be replaced with an ultranoncompliant .035-in lumen balloon (discussed previously). After initial dilation use a $4 \mathrm{~F} / 5 \mathrm{~F}$ hydrophilic catheter to exchange the 0.018-in for a 0.035-in Amplatz wire. Boston Scientific Sterling OTW (0.018-in wire lumen) Balloon Catheter: 6-mm $\times$ 40-mm balloon; shaft length 80-cm (UPN \# H74939032604080, catalog \# 39,032-60,408).

\section{FOCUSED FORCE VENOPLASTY}

Approximately $3 \%$ of cases require focused force venoplasty for a stenosis (see Fig. 7) refractory to an ultranoncompliant balloon (RBP 30 atm). Focused force can also be attempted when there is excessive elastic recoil (ie, when it remains difficult to manipulate the catheter after initial balloon dilation despite no visible waist). To apply focused force, remove the balloon, advance a long $5 \mathrm{~F}$ sheath or a $6 \mathrm{~F}$ multipurpose guide (nondiagnostic catheter) over the Amplatz wire, advance a second Amplatz wire through the sheath/guide, remove the 

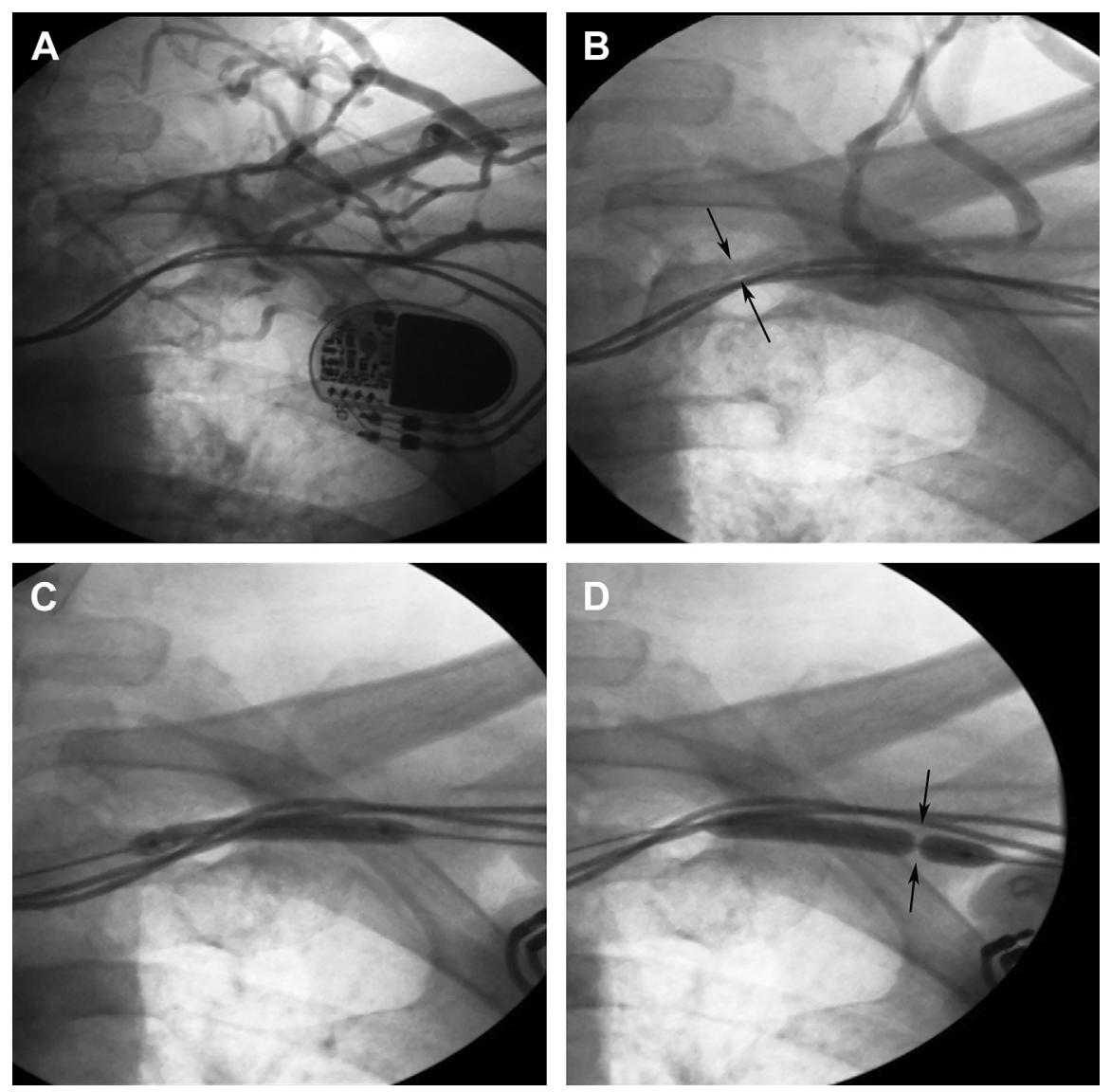

Fig. 4. Crossing a subclavian occlusion and performing venoplasty. $(A)$ The peripheral venogram reveals extensive collaterals, suggesting total occlusion. $(B)$ Injection at the site of occlusion through the dilator from a $5 \mathrm{~F}$ sheath (local venogram) reveals a stenosis (arrrows) not a total occlusion through which the wire is easily advanced. (C) A 6-mm by 4-cm noncompliant balloon is inflated at the site of occlusion seen on the venogram. $(D)$ The balloon is inflated more peripherally, revealing a stenosis (arrows) not appreciated on either the peripheral or local venogram.

sheath/guide and advance the balloon over 1 of the wires. The balloon is then inflated to RBP against the wire beside the balloon. The stiff wire beside the balloon pressed against the fibrous tissue is more aggressive then the balloon alone. The procedure is also referred to as the poor man's cutting balloon. The authors have not observed lead damage with focused force venoplasty.

\section{FACTORS THAT MAKE A SUBCLAVIAN OCCLUSION DIFFICULT TO CROSS}

An attempt at venous access in a patient with in situ lead(s) before a venogram is often successful. If there is an obstruction, however, the initial blind attempt(s) can disrupt the venous anatomy making it impossible to cross the occlusion.
If the artery is entered inadvertently the subsequent hematoma compresses the vein, making access even more difficult. If a still image of the venogram or ultrasound is started with, it is not clear where the needle enters the vein relative to the obstruction. If the needle enters the vein at the site of occlusion, the wire does not advance and/or an existing opening is obscured.

\section{COMBINING RETAINED WIRE LEAD REMOVAL AND VENOPLASTY}

In some cases, it may be impossible to cross a total subclavian obstruction. If 1 of the leads is mobile within the fibrous adhesions, retained wire lead removal followed by venoplasty can be used instead of extraction. When a lead is 

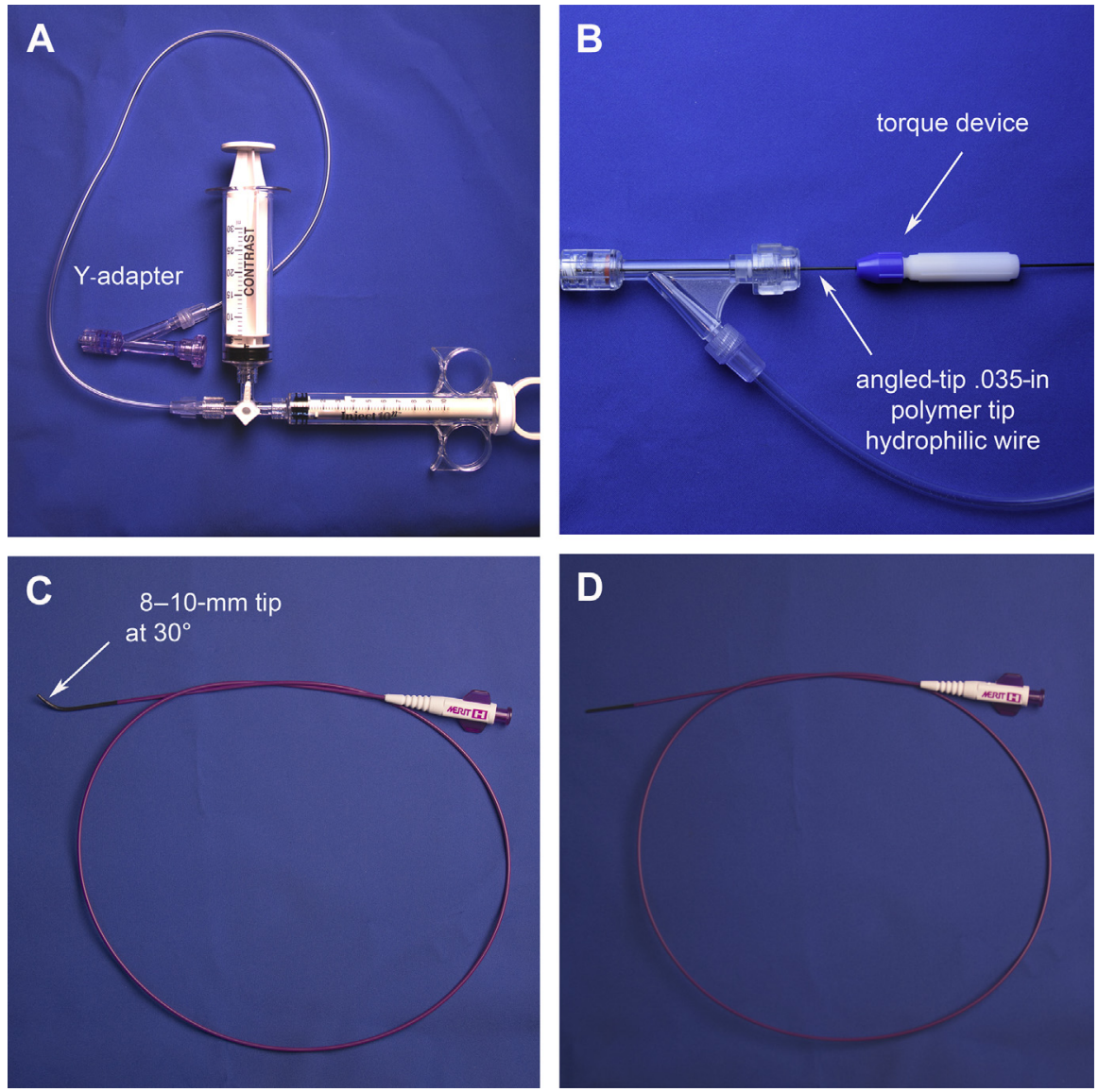

Fig. 5. Equipment used to cross and dilate a subclavian obstruction. ( $A$ ) The contrast injection system (Worley Advanced Kit 1 CAK 1 [includes contrast bowl and labels], order \# K12-WORLEY1, Merit Medical). (B) The .035 -in $\times 180-\mathrm{cm}$ angled polymer tip hydrophilic (Laureate wire, order \# LWSTDA35180, Merit Medical) is advanced through the hemostatic valve of the $\mathrm{Y}$-adapter. A torque device is essential for directing the wire through the opening observed with local contrast injection. (C) The 5F braided angled-tip hydrophilic catheter (5F 65-cm Impress KA2 Hydrophilic Angiographic Catheter, order \# 56538KA2-H, Merit Medical) is used to direct a wire through an occlusion (crossing catheter). The wire braid in the catheter provides torque control and pushability that are important for crossing difficult obstructions. The length $(6-8 \mathrm{~mm})$ and angle $\left(30^{\circ}\right)$ of the tip are also important. In my experience, similar catheters with a longer tip or more acute angle are less effective. $(D)$ The $4 \mathrm{~F}$ braided straight catheter $(65 \mathrm{~cm}$; Impress Straight Hydrophilic Angiographic Catheter, order \# 46538STS-H, Merit Medical) used to exchange an existing wire for a more supportive wire over which to advance the balloon. The authors find that a braided hydrophilic catheter advances through an obstruction when a nonbraided catheter of the same French size does not advance. (Courtesy of Merit Medical, South Jordan, UT; with permission.)

"extracted" for venous access, the fibrous adhesions encasing the lead are pulverized by the mechanical/powered sheaths to provide sufficient lumen to add a lead(s). Because of the risks involved, SVC extraction with powered/mechanical sheaths requires termination of the procedure to arrange for operating room backup. When the lead can be used to get a wire into the central circulation, venoplasty is a safer alternative to pulverization. The wire under the insulation technique is one of several methods whereby a lead can be sacrificed to introduce a wire into the central circulation (Fig. 9; see Video 7). Whether a lead can be advanced back into the circulation with a wire attached depends on the following: (1) the surface characteristic of the pacing lead insulation (ie, silicone, polyurethane, or copolymer hybrid); (2) the characteristics of the fibrous binding (ie, thickness, elasticity, density, and adhesion of the lead); (3) the length of the fibrous binding surrounding the lead (ie, left greater than right); 

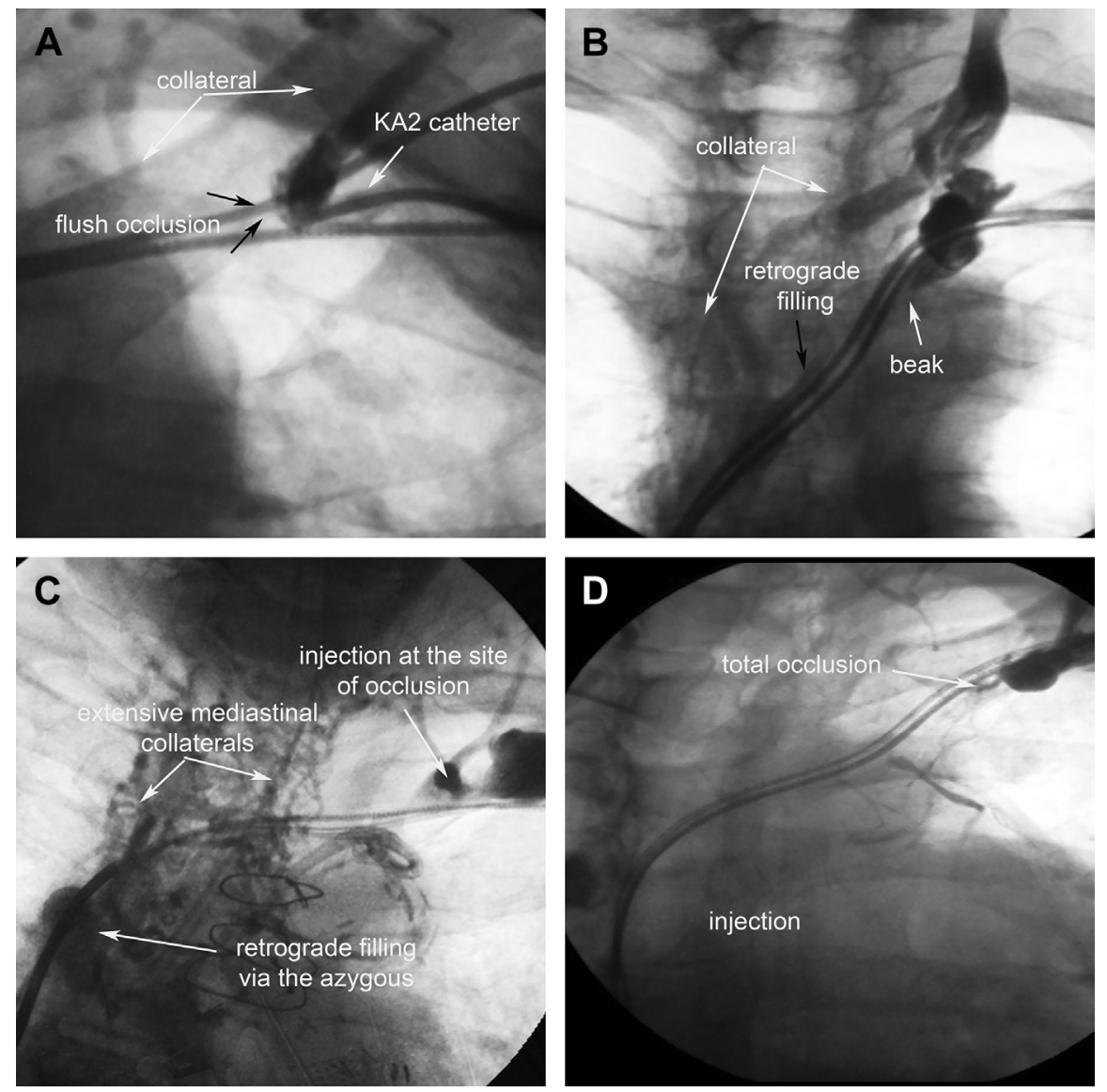

Fig. 6. Subclavian occlusion despite injection at the site of occlusion (local venogram). $(A)$ Injection with the angled tip hydrophilic braided crossing catheter (see Fig. 5C) at the site of obstruction reveals that the subclavian is flush occluded. Despite this the obstruction was crossed using the KA2 catheter and glide wire. The KA2 was advanced into the RA, the hydrophilic wire exchanged for an extra support wire and venoplasty performed. $(B)$ Injection at the site of occlusion reveals near-total occlusion; however, contrast is seen to taper to a beak shaped. Directing the wire into the beak with the KA2 is associated with a good chance of successful crossing of the occlusion. Retrograde filling of contrast is also observed and is associated with successful crossing. The KA2 and glide wire were used to successfully cross the occlusion. (C) There is total occlusion at the site of injection though the dilator from a $5 \mathrm{~F}$ sheath. There is retrograde filling via collateral to the azygous vein. A beak at the site of occlusion suggests that crossing the occlusion is possible. Surprisingly, a .035-in angled polymer tip hydrophilic wire was easily passed through the occlusion without the need for the KA2. The glide wire was exchanged for a .035-in superstiff wire using the 4F braided hydrophilic exchange catheter (see Fig. 5D) but the 6- $\mathrm{mm}$ diameter balloon did not advance through the occlusion. The .035-in wire was exchanged for a .018-in wire and a 6-mm $\times$ 4-cm low-profile balloon was advanced through the occlusion and venoplasty performed. $(D)$ Total occlusion without a beak is seen with contrast injection at the site of occlusion. There is no retrograde filling of the innominate. Femoral access and injection at the site where the lead enters the SVC revealed flush occlusion. The obstruction could not be crossed.

(4) the diameter of the wire attached to the lead because a larger wire is less likely to fit in the fibrous sleeve beside the pacing lead than a smaller wire; and (5) the friction generated between the surface of the wire and the fibrous binding. A wire with a polymer jacket (ie, the black coating on a glide wire) has a lower coefficient of friction than a standard hydrophilic wire. Polymer jacketed wires are commonly referred to simply as hydrophilic, which can cause confusion.

When the lead does not advance back into the central circulation, access can be salvaged by cutting off the connector pin end and inserting the wire into the stylet lumen. The lead is then extracted from below with a loop snare pulling 


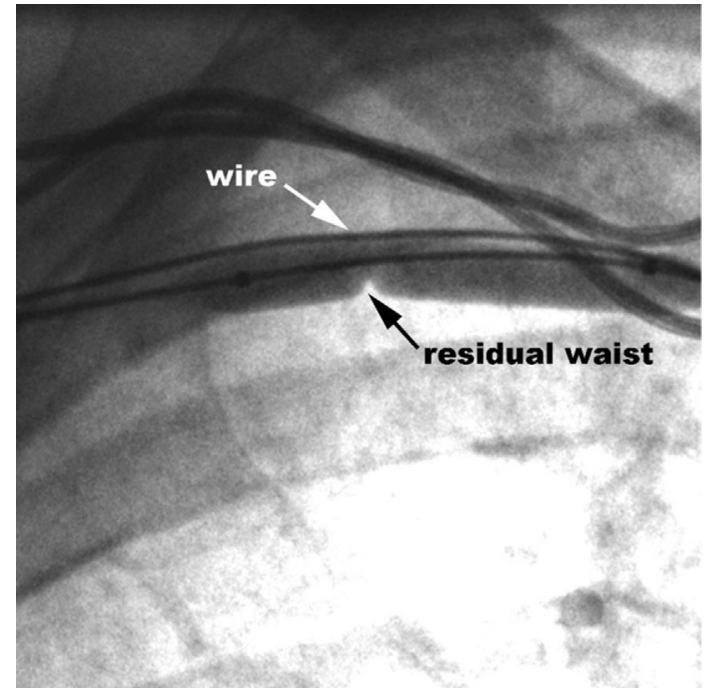

Fig. 7. Focused force venoplasty. See text for details. the lead and wire into the central circulation (Fig. 10). Modern LV pacing leads can frequently be removed over a wire. Without venoplasty, however, the fibrous adhesions make it difficult/ impossible to use the retained wire to add a lead. When the LV lead is movable but the wire does not exit the tip, it can be snared and removed from below pulling the wire into the central circulation. Pulling the lead from below can also be useful when the insulation bunches up (ie, accordion effect). Combining femoral lead removal of an existing lead with venoplasty can avoid the need for surgical backup and is a particularly attractive alternative in patients with prior sternotomy. For example, if there is an existing active fixation atrial lead in a patient with prior open heart surgery, the lead can be used for access, as illustrated in Fig. 10. Conversely, retained wire femoral
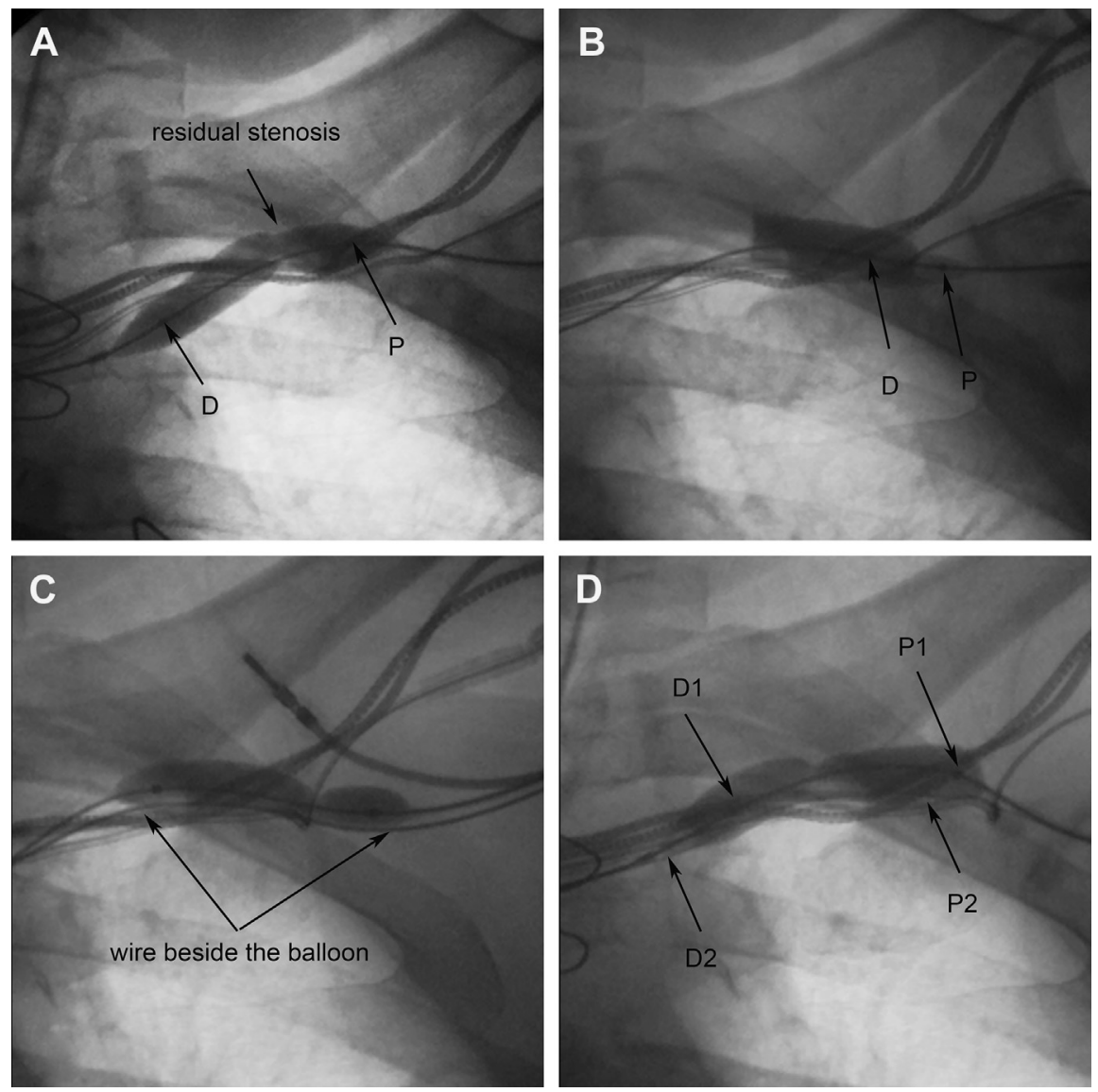

Fig. 8. Difficult subclavian obstruction with extensive elastic recoil. $(A)$ The balloon is inflated with a residual waist. The distal (D) and proximal (P) markers on the balloon catheter are separated by $4 \mathrm{~cm}$. (B) The balloon is withdrawn before the contrast is fully removed. The proximal $(P)$ and distal $(D)$ markers on the balloon are closer together indicating that the tip of the balloon is folded back on itself making it larger and more difficult to remove. (C) After the balloon is readvanced a wire is advanced beside the balloon and the balloon inflated against the wire (focused force venoplasty). (D) A second balloon is advanced beside the first and both balloons inflated simultaneously. D1 and P1 are the distal and proximal markers, respectively, on the first balloon. D2 and $\mathrm{P} 2$ are the distal and proximal markers, on the second balloon. 
A
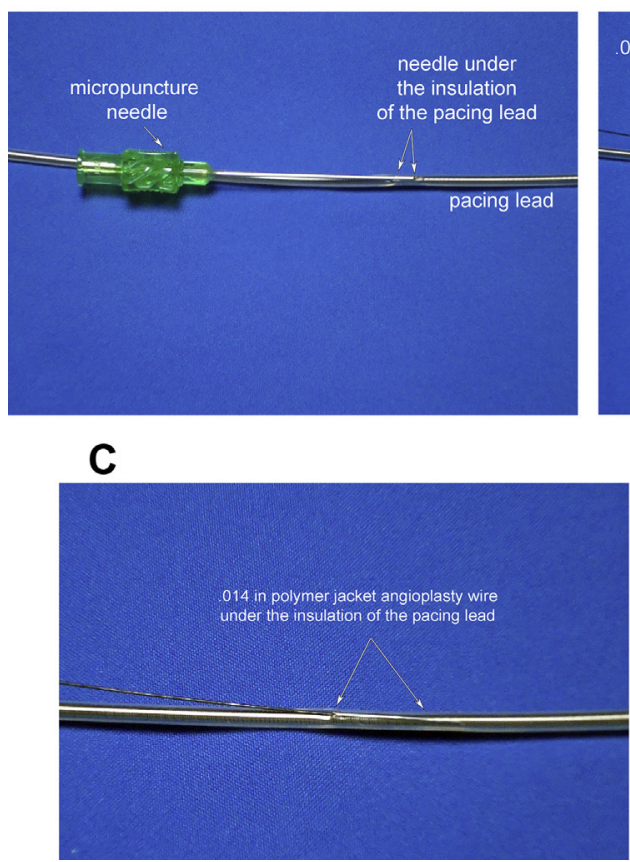

B

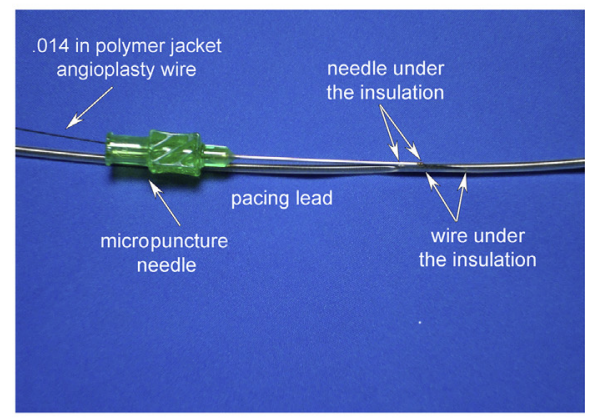

D

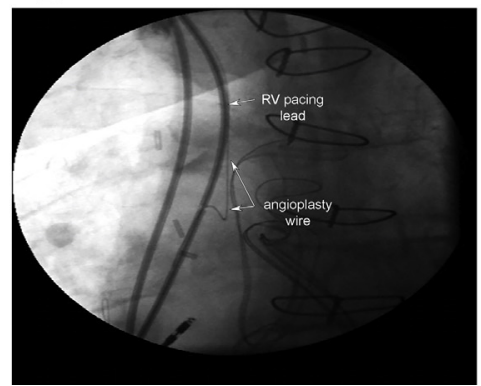

Fig. 9. Wire under the insulation technique for retained wire lead removal followed by venoplasty. $(A)$ The tip of the micropuncture needle is inserted under the insulation of the pacing lead. (B) A polymer jacketed extrastiff .014-in wire is advanced through the micropuncture needle between the insulation and the outer conductor of the pacing lead. (C) The needle is removed with the wire under the insulation. (D) The pacing lead is advanced back into the circulation with the wire attached.

extraction of an old passive fixation atrial lead in a patient without previous open heart surgery could result in perforation at the site of attachment of the tip of the lead to the myocardium.

\section{SUBCLAVIAN VENOPLASTY: QUALITY OF VENOUS ACCESS, LEAD BURDEN, AND PRESERVATION OF VENOUS ACCESS}

The 2017 HRS Expert Consensus statement ${ }^{29}$ includes SV as an option when venous access becomes an issue due to occlusion of the desired access point. The discussion does not address the quality of venous access provided by SV compared with progressively larger dilators. Restricted catheter manipulation from residual stenosis compromises the implanters ability to implant leads, particularly evident with His bundle pacing and LV lead implantation.

In addition, the Consensus Statement suggests that venoplasty adds to overall lead burden by leaving redundant lead(s) behind. This is not the case when venoplasty is used for access in the absence of redundant leads or when used in conjunction with the retained wire lead removal. Because of the risk and logistics associated with extraction, many implanting physicians, when faced with a subclavian obstruction, implant an entirely new system on the contralateral side. Adding SV as an option certainly preserves venous access and likely reduces lead burden even when performed with a redundant lead, for example, upgrade from pacemaker to ICD. With regard to preservation of venous access, the expert consensus seems to equate SV to contralateral lead implantation with tunneling across the chest. Unlike tunneling, SV preserves venous access. SV should be the standard of care for access in most patients with venous occlusion without redundant leads. Recognizing that extraction, unlike SV, results in (1) procedural delay while operating room backup is arranged, (2) early reopening of the pocket with increased risk of infection, (3) higher expense, and (4) increased risk of minor and major complications, which calls into question the use of extraction as the first-line approach to device upgrades for patients with venous occlusion and redundant leads, even when performed in experienced centers. Centers experienced in extraction can easily add SV to their lead management options. 

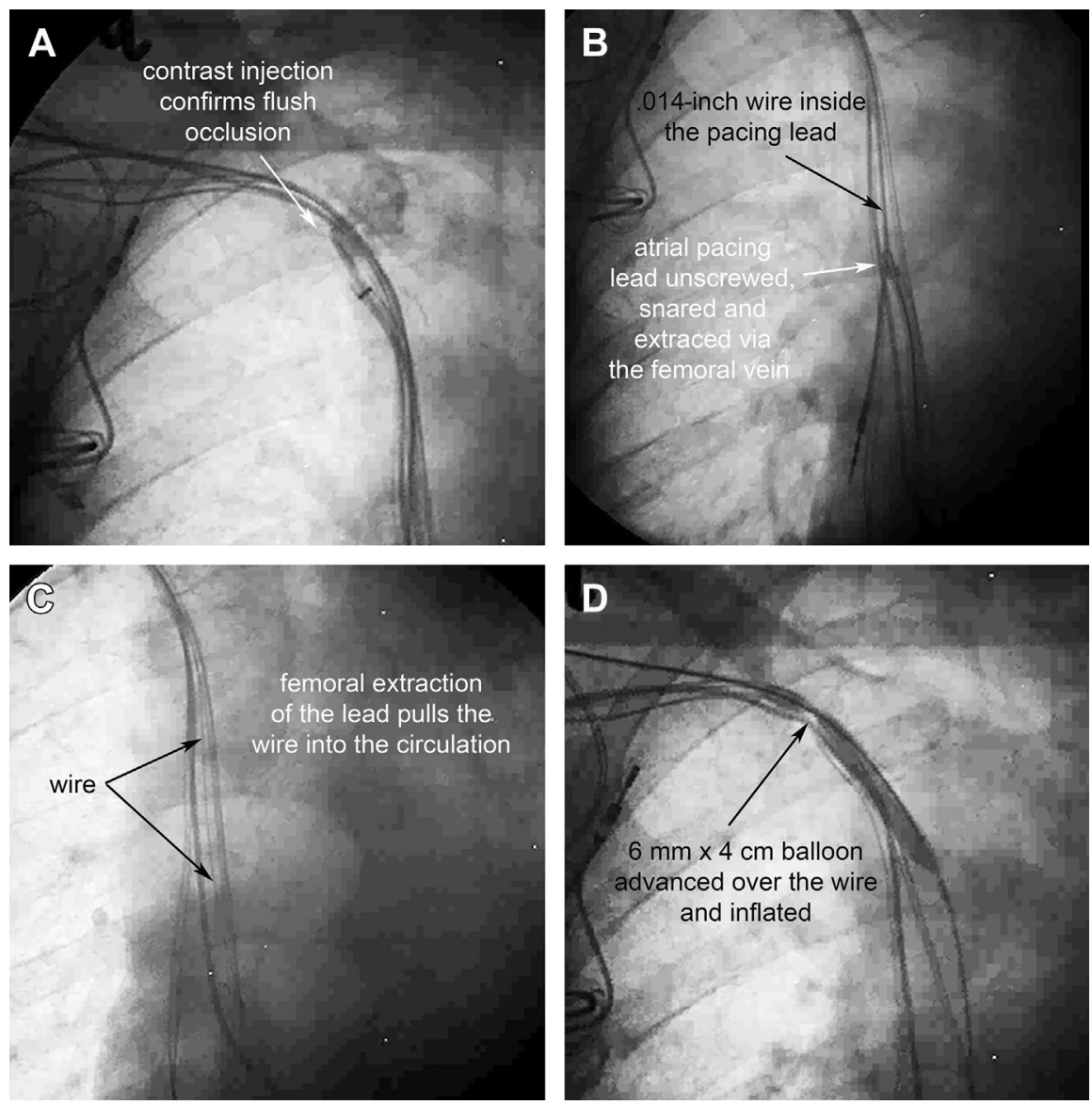

Fig. 10. Retained wire femoral lead removal followed by venoplasty in a case of total occlusion that could not be crossed. (A), femoral access and contrast injection confirms that the right innominate is flush occluded at the SVC. It was not possible to advance a wire from either direction despite using the braided angled hydrophilic catheter (KA2). (B) The suture sleeve of the active fixation atrial lead was released and the screw retracted in an unsuccessful attempt to use the wire under the insulation technique to advance a wire into the circulation. Subsequently the IS-1 connector was removed and a 300-cm 014-in angioplasty wire advanced through the stylet lumen to the tip of the lead. A $25-\mathrm{mm}$ loop snare $(6 \mathrm{~F} \times 100-\mathrm{cm}$ snare catheter + a loop 25-mm diameter $120-\mathrm{cm}$ in length One Snare, order \# ONE2500, Merit Medical) was advanced through a long sheath over the tip and closed on the body of the atrial lead containing the angioplasty wire. $(C)$ The atrial lead was extracted via the femoral vein pulling the angioplasty wire into the circulation. The .014-in angioplasty wire was exchanged for a .035-in superstiff wire using the $4 \mathrm{~F}$ braided hydrophilic exchange catheter (see Fig. 5D). (D) A $6 \mathrm{~mm} \times 4 \mathrm{~cm}$ noncompliant balloon was advanced to the subclavian-SVC junction and inflated to the RBP of the balloon. Overlapping balloon inflations were performed until the tail of the balloon was seen in the pocket on the final inflation.

\section{SUPPLEMENTARY DATA}

Supplementary data related to this article can be found online at https://doi.org/10.1016/j.ccep. 2017.11.017.

\section{REFERENCES}

1. Sticherling C, Chough SP, Baker RL, et al. Prevalence of central venous occlusion in patients with chronic defibrillator leads. Am Heart J 2001;141(5): 813-6.
2. Lickfett L, Bitzen A, Arepally A, et al. Incidence of venous obstruction following insertion of an implantable cardioverter defibrillator. A study of systematic contrast venography on patients presenting for their first elective ICD generator replacement. Europace 2004;6(1):25-31.

3. Spittell PC, Vlietstra RE, Hayes DL, et al. Venous obstruction due to permanent transvenous pacemaker electrodes: treatment with percutaneous transluminal balloon venoplasty. Pacing Clin Electrophysiol 1990;13(3):271-4. 
4. Spittell PC, Hayes DL. Venous complications after insertion of a transvenous pacemaker. Mayo Clin Proc 1992;67(3):258-65.

5. Oginosawa Y, Abe H, Nakashima Y. The incidence and risk factors for venous obstruction after implantation of transvenous pacing leads. Pacing Clin Electrophysiol 2002;25(11):1605-11.

6. Haghjoo M, Nikoo MH, Fazelifar AF, et al. Predictors of venous obstruction following pacemaker or implantable cardioverter-defibrillator implantation: a contrast venographic study on 100 patients admitted for generator change, lead revision, or device upgrade. Europace 2007;9(5):328-32.

7. Bulur S, Vural A, Yazıcı M, et al. Incidence and predictors of subclavian vein obstruction following biventricular device implantation. J Interv Card Electrophysiol 2010;29(3):199-202.

8. Bracke F, Meijer A, Van Gelder B. Venous occlusion of the access vein in patients referred for lead extraction: influence of patient and lead characteristics. Pacing Clin Electrophysiol 2003;26(8):1649-52.

9. Poole JE, Gleva MJ, Mela T, et al. Complication rates associated with pacemaker or implantable cardioverter-defibrillator generator replacements and upgrade procedures: results from the REPLACE registry. Circulation 2010;122(16):1553-61.

10. Duray GZ, Israel CW, Pajitnev D, et al. Upgrading to biventricular pacing/defibrillation systems in right ventricular paced congestive heart failure patients: prospective assessment of procedural parameters and response rate. Europace 2008;10(1):48-52.

11. McCotter CJ, Angle JF, Prudente LA, et al. Placement of transvenous pacemaker and ICD leads across total chronic occlusions. Pacing Clin Electrophysiol 2005;28(9):921-5.

12. Worley SJ, Gohn DC, Pulliam RW, et al. Subclavian venoplasty by the implanting physicians in 373 patients over 11 years. Heart Rhythm 2011;8(4):526-33.

13. Worley SJ. Implant venoplasty: dilation of subclavian and coronary veins to facilitate device implantation: indications, frequency, methods, and complications. J Cardiovasc Electrophysiol 2008;19(9):1004-7.

14. Worley SJ, Gohn DC, Pulliam RW. Over the wire lead extraction and focused force venoplasty to regain venous access in a totally occluded subclavian vein. J Interv Card Electrophysiol 2008;23(2):135-7.

15. Baerlocher MO, Asch MR, Myers A. Successful recanalization of a longstanding complete left subclavian vein occlusion by radiofrequency perforation with use of a radiofrequency guide wire. J Vasc Interv Radiol 2006;17(10):1703-6.

16. Worley SJ, Gohn DC, Pulliam RW. Excimer laser to open refractory subclavian occlusion in 12 consecutive patients. Heart Rhythm 2010;7(5):634-8.
17. Antonelli D, Freedberg NA, Rosenfeld T. Lead insertion by supraclavicular approach of the subclavian vein puncture. Pacing Clin Electrophysiol 2001; 24(3):379-80.

18. Fox DJ, Petkar S, Davidson NC, et al. Upgrading patients with chronic defibrillator leads to a biventricular system and reducing patient risk: contralateral LV lead placement. Pacing Clin Electrophysiol 2006; 29(9): 1025-7.

19. Jones SO, Eckart RE, Albert CM, et al. Large, singlecenter, single-operator experience with transvenous lead extraction: outcomes and changing indications. Heart Rhythm 2008;5(4):520-5.

20. Byrd CL, Wilkoff BL, Love CJ, et al. Clinical study of the laser sheath for lead extraction: the total experience in the United States. Pacing Clin Electrophysiol 2002;25(5):804-8.

21. Wilkoff BL, Byrd CL, Love CJ, et al. Pacemaker lead extraction with the laser sheath: results of the pacing lead extraction with the excimer sheath (PLEXES) trial. J Am Coll Cardiol 1999;33(6):1671-6.

22. Venkataraman G, Hayes DL, Strickberger SA. Does the risk-benefit analysis favor the extraction of failed, sterile pacemaker and defibrillator leads? J Cardiovasc Electrophysiol 2009;20(12):1413-5.

23. Ji SY, Gundewar S, Palma EC. Subclavian venoplasty may reduce implant times and implant failures in the era of increasing device upgrades. Pacing Clin Electrophysiol 2012;35(4):444-8.

24. Stoney WS, Addlestone RB, Alford WC, et al. The incidence of venous thrombosis following longterm transvenous pacing. Ann Thorac Surg 1976; 22(2): 166-70.

25. Antonelli D, Turgeman Y, Kaveh Z, et al. Short-term thrombosis after transvenous permanent pacemaker insertion. Pacing Clin Electrophysiol 1989;12(2): 280-2.

26. Goto Y, Abe T, Sekine S, et al. Long-term thrombosis after transvenous permanent pacemaker implantation. Pacing Clin Electrophysiol 1998;21(6): 1192-5.

27. Da Costa SS, Scalabrini Neto A, Costa R, et al. Incidence and risk factors of upper extremity deep vein lesions after permanent transvenous pacemaker implant: a 6-month follow-up prospective study. Pacing Clin Electrophysiol 2002;25(9):1301-6.

28. Robboy SJ, Harthorne JW, Leinbach RC, et al. Autopsy findings with permanent pervenous pacemakers. Circulation 1969;39(4):495-501.

29. Kusumoto FM, Schoenfeld MH, Wilkoff BL, et al. 2017 HRS expert consensus statement on cardiovascular implantable electronic device lead management and extraction. Heart Rhythm 2017; 14(12):e503-51. 